\title{
Performance analysis of various filters for De-noising of Handwritten Kannada documents
}

\author{
Mamatha H.R \\ Assoc. Prof, Department of \\ ISE, PES Institute of \\ Technology, Bangalore, India
}

\author{
Sonali Madireddi \\ Department of CSE, PES \\ School of Engineering, \\ Bangalore, India
}

\author{
Srikanta Murthy $\mathrm{K}$ \\ Professor, Department of CSE, \\ PES School of Engineering, \\ Bangalore, India
}

\begin{abstract}
Image enhancement is used to improve the quality of digital images, when the knowledge about the source of the distortion is unknown. Image enhancement techniques are important for visual inspection and for machine analysis of a system. Handwritten scripts are prone to noise induction during image transmission where noise can be introduced by the medium of transfer. In this paper we made an attempt to undertake the study of four types of noise induced in the handwritten Kannada documents and their removal using seven types of filters. The comparative study is conducted with the help of seven different image quality measures. The images are also tested with different Gaussian noise densities ranging from $20 \%$ to $100 \%$. From the experimental results it is seen that median, average and wiener filters perform better compared to Laplacian, Canny, Robert and Prewitt. It is also observed that median filter is better in removing salt and pepper noise .The wiener and average filters are best to remove Gaussian and Poisson noise. Speckle noise can be better removed by Laplacian filter.
\end{abstract}

\section{General Terms}

Image EnhancementTechniques, Document Analysis.

\section{Keywords}

Filters, Noise, image quality measures, handwritten Kannada document.

\section{INTRODUCTION}

The Document analysis algorithms such as page segmentation and character recognition, for example, often work best on the assumption of a clean document and use principle of connected components as basic units. Unfortunately, noise often interferes with these assumptions [1].Cleanly scanned textual documents are almost ideal models. Photocopied or faxed documents with non uniform illumination and manmade smeary spots noises are very difficult to process and makes the text unreadable thus reducing the recognition accuracy. Therefore, it is necessary to perform some preprocessing to remove various noises from the document image before a recognition algorithm is applied.[2]

Handwritten scripts are affected with different types of noise during transmission such as Gaussian noise, Salt \& Pepper Noise, Poisson noise, Speckle noise. The handwritten images may get affected by distortions and deformities due to time where the script will get faded and will develop a low contrast and a blurry image. To remove the disadvantage of viewing the document which has been affected with time and also by transmission impairments, image filtering and enhancement techniques are very important. To understand how we can better enhance the handwritten text, this paper assess seven different filtering algorithms on four types of noise which can affect the hand written Kannada documents and estimates the filter's performance by using seven varied types of image quality measures. The filtering algorithms are implemented on twenty five samples for various noise types. The simulation is performed on MATLAB R2007b version.

Noise removal is easier in the spatial domain as compared to the frequency domain as the spatial domain noise removal requires very less processing time [3].Thus this paper intends to perform noise removal in the spatial domain rather than use frequency Fourier transforms. This present work focuses on image de-noising approaches applied to handwritten Kannada scripts. Old handwritten Kannada scripts have to be maintained so that future generations can access the ancient works. Vast amounts of historical hand written texts are the property of state and country libraries, where these texts will be converted to their digital form [4] to preserve the information in secondary sources even if the primary sources such as ancient scrolls of text get degraded. Handwritten documents may be inflicted with much deformation and degradation such as seepage of ink, presence of ink blots, smears and stains [4].Hence it is imperative to develop noise removal techniques to act on handwritten text. Higher document understanding, efficient analysis, improved character segmentation are some of the advantages of noise removal from document images [2].

The past research work emphasized on removing noise from remote sensing images [5],Binary document images [1], underwater images [6].In this paper we made an attempt to undertake the study of four types of noise in the handwritten Kannada documents and their removal using seven types of filters. The comparative study is conducted with the help of seven different image quality measures.

The structuring of the paper is as follows: section 2 describes the types of noise which will be removed by the mentioned filters, section 3 describes the types of filters used, section 4 describes the experimental results and discussions followed by section 5 highlighting the conclusion and section 6 lists the references.

\section{NOISE TYPES}

Noise in documents is classified based on the criteria if it is dependent on the underlying content or independent of the underlying content. Stray marks, marginal noise, ink blobs and salt-and-pepper noise are independent of size; location of the underlying content [1].Similarly the texture of the observed speckle pattern is independent of the underlying content. Blur, pixel-shift or bleed-through on other hand is dependent noise, as they manifest themselves differently depending on the content. Such content-dependent noise is comparatively more difficult to model, mathematically nonlinear and often multiplicative.

Noise can also be classified based on its consistency in properties like periodicity of occurrence in the document, its shape, position and gray-values. If noise shows a consistent 
behaviour in terms of these properties, it is called regular noise. Examples Unwanted punched holes, stray marks and ruled lines. On the other hand, noise such has ink blobs, complex background binarized pattern marginal noise and salt-and-pepper often lack a consistent property and are classified under 'irregular noise' [1]. In this paper we have made an attempt to study the four common types of noises like Gaussian, salt and pepper, Poisson and speckle noise.

\subsection{Gaussian Noise}

Gaussian noise also called Random Variation Impulsive Noise (RVIN) or normal noise $\mathrm{T}$ is a type of statistical noise in which the amplitude of the noise follows that of a Gaussian distribution. Gaussian Noise occurs as the probability density function of the normal distribution. Thus Gaussian Noise represents the frequency spectrum that has a bell shaped curve.

Gaussian distribution noise can be expressed by:

$$
\begin{aligned}
& P(x)=1 /(\sigma \sqrt{2} \pi) * e^{(x-\mu)^{2}} / 2 \sigma^{2} \text { For }-\infty \text { to } \\
& +\infty
\end{aligned}
$$

Where: $\mathrm{P}(\mathrm{x})$ is the Gaussian distribution noise in image; $\mu$ and $\mathrm{s}$ is the mean and standard deviation respectively.

\subsection{Salt-and-pepper Noise}

Salt-and-pepper noise is also called as Fat-tail distributed or impulsive noise or spike noise. An image containing salt-andpepper noise will have dark pixels in bright regions and bright pixels in dark regions. Salt and pepper noise is predominantly found in digital transmission and storage. It can be described as:

$I(t)=(1-e) S(t)+e N(t)$

$\mathrm{S}(\mathrm{t})$ represents the amount of dark pixels in bright regions, $N(t)$ represents bright pixels in dark regions and $I(t)$ represents the overall salt-and-pepper noise in the given image and $\mathrm{e}=\{0,1\}$, with a probability $\mathrm{P}$. There is a clear $50 \%$ probability of the occurrence of either black or white pixels within the image giving rise to salt and pepper noise.

\subsection{Poisson Noise}

Statistical Quantum Fluctuations induce a prominent noise type in the lighter parts of an image from an image sensor. This noise is called photon shot noise or Poisson noise. The noises at different pixels are independent of each other. Poisson noise follows a Poisson distribution. The images which are obtained from radiography are mostly the ones that satisfy a Poisson distribution. The dark leakage current of the image sensor will produce yet another type of noise called the "dark current shot noise"

\subsection{Speckle Noise}

Speckle noise is a granular noise that increases the mean grey level of a local area in a image. This type of noise makes it difficult for image recognition and interpretation. In this noise type, the sample mean and variance of a single pixel is proportional to that of the mean and variance of the local area that is centred on that pixel. It is a deterministic, random and consists of an interference pattern.

\section{IMAGE FILTERING ALGORITHMS}

In image processing, filters are mainly used to suppress either the high frequencies in the image, i.e. smoothing the image, or the low frequencies, i.e. enhancing or detecting edges in the image. Image restoration and enhancement techniques are described in both the spatial domain and frequency domain, i.e. Fourier transforms. Noise removal is easier in the spatial domain as compared to the frequency domain as the spatial domain noise removal requires very less processing time [3].

Spatial processing is classified into point and mask processing. Point processing involves the transformation of individual pixels independently of other pixels in the image. These simple operations are typically used to correct for defects in image acquisition hardware, for example to compensate for under/over exposed images. On the other hand, in mask processing, the pixel with its neighbourhood of pixels in a square or circle mask are involved in generating the pixel at $(\mathrm{x}, \mathrm{y})$ coordinates in the enhanced image. It is a more costly operation than simple point processing, but more powerful. The application of a mask to an input image produces an output image of the same size as the input.

One of the most important requirement of noise removal algorithms is that they should provide satisfactory amount of noise removal and also help preserve the edges. For the stated conditions to be satisfied, there are two types of filters with their significant advantages and disadvantages. The two types of filters are the linear and non-linear filters. The linear filters have the advantage of faster processing but the disadvantage of not preserving edges. Conversely the non-linear filters have the advantage of preserving edges and the disadvantage of slower processing [7]. Thus this paper intends to perform noise removal in the spatial domain rather than use frequency Fourier transforms.

\subsection{Median Filter}

It is important to perform noise removal during signal processing on an image or on a signal. One of the methods to perform this noise reduction is by performing neighbourhood averaging. The neighbourhood averaging can suppress isolated out-of-range noise, but the side effect is that it also blurs sudden changes such as sharp edges. The median filter is an effective method that can suppress isolated noise without blurring sharp edges. In Median Filtering, all the pixel values are first sorted into numerical order and then replaced with the middle pixel value. [8]

Let $\mathrm{y}$ represent a pixel location and $\mathrm{w}$ represent a neighbourhood centred around location $(m, n)$ in the image, then the working of median filter is given by

$y[m, n]=\operatorname{median}\{x[i, j],(i, j)$ belongs to $w\}$

Since the pixel $y[m, n]$ represents the location of the pixel $y$ , $\mathrm{m}$ and $\mathrm{n}$ represents the $\mathrm{x}$ and $\mathrm{y}$ co-ordinates of $\mathrm{y}$. $\mathrm{W}$ represents the neighbourhood pixels surrounding the pixel position at $(m, n) .(i, j)$ belongs to the same neighbourhood centred around $(m, n)$.Thus the median method will take the median of all the pixels within the range of $(i, j)$ represented by $x[i, j]$

\subsection{Wiener Filter}

The inverse filtering is a restoration technique for deconvolution, i.e., when the image is blurred by a known low pass filter, it is possible to recover the image by inverse filtering or generalized inverse filtering. However, inverse filtering is very sensitive to additive noise. The approach of 
reducing degradation at a time allows us to develop a restoration algorithm for each type of degradation and simply combine them. The Wiener filtering executes an optimal trade-off between inverse filtering and noise smoothing. It removes the additive noise and inverts the blurring simultaneously.

The Wiener filtering is optimal in terms of the mean square error. In other words, it minimizes the overall mean square error in the process of inverse filtering and noise smoothing. The Wiener filtering is a linear estimation of the original image. The approach is based on a stochastic framework. The orthogonality principle implies that the Wiener filter in Fourier domain can be expressed as follows:

$$
w\left(f_{1}, f_{2}\right)=\frac{H^{*}\left(f_{1}, f_{2}\right) S_{x x}\left(f_{1} f_{2}\right)}{\left|H\left(f_{1} f_{2}\right)\right|^{2} S_{x x}\left(f_{1} f_{2}\right)+S_{\eta \eta}\left(f_{1} f_{2}\right)^{\prime}}
$$

(4)

Where $S_{x x}\left(f_{1}, f_{2}\right), S_{\eta \eta}\left(f_{1}, f_{2}\right)$ are respectively power spectra of the original image and the additive noise, and $H\left(f_{1}, f_{2}\right)$ is the blurring filter. It is easy to see that the Wiener filter has two separate part, an inverse filtering part and a noise smoothing part. It not only performs the deconvolution by inverse filtering (high pass filtering) but also removes the noise with a compression operation (low pass filtering).

\subsection{Average Filter}

Mean filtering is a simple, intuitive and easy to implement method of smoothing images, and to reduce the amount of intensity variation between one pixel and the next.

Average filtering replaces each pixel value in an image with the mean value of its neighbours, including itself. The simplest procedure would be to calculate the mask for all the pixels in the image. For all the pixels in the image which fall under this mask, it will be considered as the new pixel [7]. This has the effect of eliminating pixel values which are unrepresentative of their surroundings. Average filter is also considered to be a convolution filter or a mean filter.

\subsection{Laplacian Filter}

Detecting edges within an image can be done by the laplacian filter. It denotes areas where the intensity changes rapidly, hence producing an image with all the edges. The Laplacian is often applied to an image that has first been smoothed with something approximating a Gaussian smoothing filter, in order to reduce its sensitivity to noise. The operator normally takes a single gray level image as input and produces another grey level image as output. As radius of interest on the image is increased, this method will prove to be more computationally expensive.

\subsection{Canny Filter}

The canny filter is an edge detection operator that uses a multi-stage algorithm to detect a wide range of edges in images. The canny edge filter satisfies the properties like good detection, good localization and minimal response. In canny edge detection, the structural details of the image will be maintained while the amount of data within an image will reduce. [9]

\subsection{Prewitt Filter}

The Prewitt filter operator is used in image processing particularly within edge detection algorithms. Prewitt filter is a discrete differentiation operator computing an approximation of the gradient of the image intensity function. At each point in the image, the result of the Prewitt operator is either the corresponding gradient vector or the norm of this vector. The Prewitt operator is based on convolving the image with a small, separable, and integer valued filter in horizontal and vertical direction and is therefore relatively inexpensive in terms of computations. On the other hand, the gradient approximation which it produces is relatively crude, in particular for high frequency variations in the image. The working of Prewitt filter consists of computing the root mean square root of two 3 cross 3 matrices. [10]

\subsection{Robert Filter}

The Roberts filter is one of the oldest operators. It is one of the non-linear filters as it helps in edge detection and edge preservation. It is very easy to compute as it uses a $2 \times 2$ neighbourhood of the current pixel. The primary disadvantage of the Robert filter is its high sensitivity to noise, because very few pixels are used to approximate the gradient [11].

\section{EXPERIMENTAL RESULTS AND DISCUSSIONS}

A number of simulations and experiments have been conducted using Matlab R2009b version for evaluation of the filters on four different types of noise. In order to do this, we have collected 25 samples of the handwritten Kannada documents written by different people of various ages. The samples were scanned using HP flatbed scanner at 300 dpi. Experiments were conducted using these documents. One binary image document is illustrated to demonstrate the experimental results.

To test the accuracy of the Filtering algorithms, below stated steps are followed.

a) First an uncorrupted handwritten Kannada document image is taken as input.

b) Next the document image is converted to binary image.

c) Different noises are added to the handwritten Kannada document image artificially with $10 \%$ noise density.

d) The filtering algorithms are applied for reconstruction of handwritten Kannada document images.

e) To assess the performance of the filters for removal of noise and to estimate the quality of the reconstructed image, the 7 different image quality measures [12] are calculated.

f) To test the performance of the filters for varying noise density, Gaussian noise with different variance is applied on the binary document image.

Table 1-4 show the image quality measures of the four types of noise namely Gaussian, Poisson,Salt and Pepper noise and speckle noise.The values obtained for the image quality measures at different Gaussian noise levels for different filters are graphically represented in the figure 1-7.Figure 8(a) shows the binarized image of the original document. Figure 8(b)-8(e) shows the binarized document image added with different 
types of noise.Figure 9 and 10 depict the results after the application of Average filter and Median filter respectively.

\subsection{PSNR}

The peak Signal to Noise ratio is calculated by:

$$
P S N R=10 \log _{10}\left(\frac{255^{2}}{M S E}\right)
$$

For the image quality measures, if the value of the PSNR is very high for an image of a particular noise type then is best quality image. PSNR value results in high values for removal of both Gaussian noise and Salt-and-pepper noise by using the median filter. The average filter will remove the Gaussian Noise better followed with that of Poisson noise. According to the PSNR values, the wiener filter is best suited to remove the Gaussian Noise. According to performance comparison we see that median filter is more suited to remove the salt-andpepper noise and wiener filter is more suited to remove Gaussian and speckle noise. $20 \%$ Noise density is resulting in better image quality for all the types of filters with respect to PSNR evaluation.

\subsection{MSE values}

Mean square error is given by

$$
M S E=\frac{1}{M N} \sum_{i=1}^{M} \sum_{i=1}^{N}[g(i, j)-f(i, j)]^{2}
$$

Where $\mathrm{M}$ and $\mathrm{N}$ are the total number of pixels in the horizontal and the vertical dimensions of image, $g$ denotes the Noise image and $f$ denotes the filtered image.

The lowest mean square error represents the best quality image, thus median filter is best suited to remove the salt-andpepper noise according to this quality measure. The average and wiener filter are the best for removing Poisson noise and Gaussian noise respectively. Canny, Robert and Prewitt filter have lesser efficiency than the median, average and wiener filters but they have edge detection capability. $20 \%$ Noise density is resulting in better image quality for all the types of filters with respect to MSE evaluation.

\subsection{Average Difference}

The average difference can be found by

$$
(A D)=\frac{1}{M N} \sum_{i=1}^{M} \sum_{j=1}^{N}\left[f(i, j)-f^{\prime}(i, j)\right]
$$

A lower value of Average difference represents a better quality image [12].Hence the median filter shows better noise removal from both Gaussian noise and salt and pepper noise. Both Average filter and Wiener filter are more suited to remove Gaussian noise according to average difference measure. The Average difference measure shows that Laplacian filter is suitable for removing the speckle noise. All the 3 filters of canny, Robert and Prewitt are best suited for speckle noise as well.

\subsection{Normalized Correlation}

Normalized correlation is calculated by:

$$
(N K)=\frac{\sum_{i=1}^{M} \sum_{j=1}^{N}\left[f(i, j) \cdot f^{\prime}(i, j)\right]}{\sum_{i=1}^{M} \sum_{j=1}^{N} f(i, j)^{2}}
$$

For image-processing applications in which the brightness of the image and template can vary due to lighting and exposure conditions, the images can be first normalized. This is typically done at every step by subtracting the mean and dividing by the standard deviation. If the normalized cross correlation tends to 1 , then the image quality is deemed to be more better. This quality of better image quality is seen in the Median, average and wiener filters.

\subsection{Maximum Difference}

Maximum Difference is calculated by:

$$
(M D)=\operatorname{Max}\left(\left|f(i, j)-f^{\prime}(i, j)\right|\right)
$$

Maximum difference is used to calculate the picture quality after different compression techniques and thus it is used to measure the quality of the compressed picture quality. Large values of Maximum difference shows that the picture quality is poor.Wiener filter, Average filter results show that the final image quality is good but the Laplacian , Canny, Robert and Prewitt show that the image quality is poor due to very high Maximum difference values.

\subsection{Normalized Absolute Error}

Normalized absolute error is calculated by:

$$
(N A E)=\frac{\sum_{i=1}^{M} \sum_{j=1}^{N}\left|\left[f(i, j) \cdot f^{\prime}(i, j)\right]\right|}{\sum_{i=1}^{M} \sum_{j=1}^{N}|f(i, j)|}
$$

Normalized absolute error indicates how different both the denoised image and the original image are with the value of zero being the perfect fit. LARGE VALUES OF NAE represents poor quality of the image. Thus Median, Average and Wiener images show better quality images due to their values being very small and the Laplacian, Canny, Robert, Prewitt show bad quality images since their NAE values are very large.

\subsection{Structural Content}

Structural Content is calculated by:

$$
(S C)=\frac{\sum_{i=1}^{M} \sum_{j=1}^{N}[f(i, j)]^{2}}{\sum_{i=1}^{M} \sum_{j=1}^{N} f^{\prime}(i, j)^{2}}
$$

If the structural content value is placed at 1 , then it represents a better quality image. If the values are too large then it represents poor quality. Thus, Median, Average, Wiener filters show better quality images and the Laplacian, Canny, Robert, Prewitt show poor quality images.

\section{CONCLUSION}

In this paper seven filtering algorithms were applied on four different noise types of Gaussian, Poisson, Salt \& Pepper and 
Speckle noise which would be developed in a Kannada handwritten document during image capture, during transmission or that would have developed due to the progression of time leading to blurring and poor contrast of the written letters. Seven image performance techniques were chosen to evaluate the filters on different noise levels. From the experimental results it is seen that median, average and wiener filters perform better compared to Laplacian , Canny, Robert and Prewitt. It is also observed that median filter is better in removing salt and pepper noise .The wiener and average filters are best to remove Gaussian and Poisson noise. Speckle noise can be better removed by Laplacian filter.

\section{REFERENCES}

[1] MuditAgrawal and David Doermann,"Clutter noise removal in Binary Document Images".Published in Document Analysis and Recognition,2009.ICDAR '09.10th International conference.

[2] ZangPing,ChenLihui,"Document filters using morphological and geometrical features of characters",Image and Vision Computing,Elsevier 2001,19(2001)847-855

[3] Nichol, J.E. and Vohra, V., Noise over water surfaces In Landsat TM images, International Journal of Remote Sensing, Vol.25, No.11, 2004, PP.2087 - 2093.

[4] ErginaKavallieratou and EfstathiosStamatatos,"Improving the quality of Degraded document images",Proceedings of the Second International Conference on Document Image Analysis for Libraries (DIAL'06)

[5] Mr. Salem Saleh Al-amri, Dr. N.V. Kalyankar and Dr. Khamitkar S.D ,"A comparative study of removal noise from remote sensing image" Published by IJCSI International Journal of Computer Science Issues, Vol. 7, Issue. 1, No. 1, January 2010.

[6] Dr.G.Padmavathi, Dr.P.Subashini, Mr.M.Muthu Kumar and Suresh Kumar Thakur,"Comparison of filters used for underwater Image-Preprocessing",IJCSNS International Journal of Computer Science and Network Security, VOL.10 No.1, January 2010

[7] PawanPatidar,ManojGupta,SumitSrivastava,Ashok Kumar Nagawat,"Image de-noising by various filters for different noise",Published in International Journal of computer applications, Vol-9,November 2010

[8] Y.Murali Mohan Babu, Dr.M.V. subramanyam, Dr.M.N.Giri Prasad," PCA based image denoising", Published in SIPIJ,Vol 2 ,April 2012.

[9] MasoudNosrati, RonakKarimi,Mehdi Hariri," Detecting circular shapes from areal images using median filter and CHT", Published in Global Jounal of Computer Science and Technology.Volume 12,January 2012

[10] Parminder Singh and Harjinder Singh “ A comparison of High Pass Spatial Filters using Measurements and Automation", Published in IJERT,Vol 1 May 2012

[11] Sonka,Hlavac,Boyle,'Digital Image Processing and Computer Vision",Cengage Learning

[12] Sumathi Poobal,G.Ravindran,"The performance of fractal image compression on different imaging modalities using objective quality measures". International Journal of Engineering Science and Technology (IJEST).

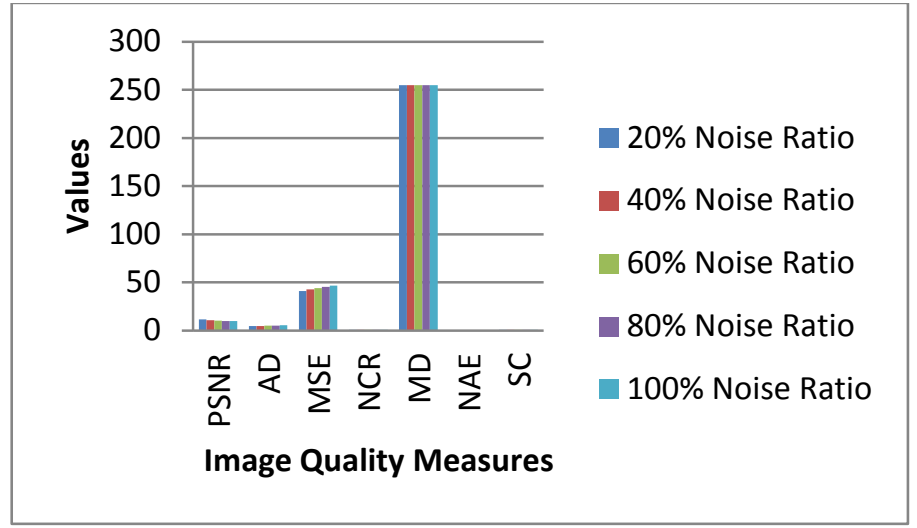

Fig 1: Graphical Representation of Image Quality Measures at different Gaussian noise levels for Median Filter

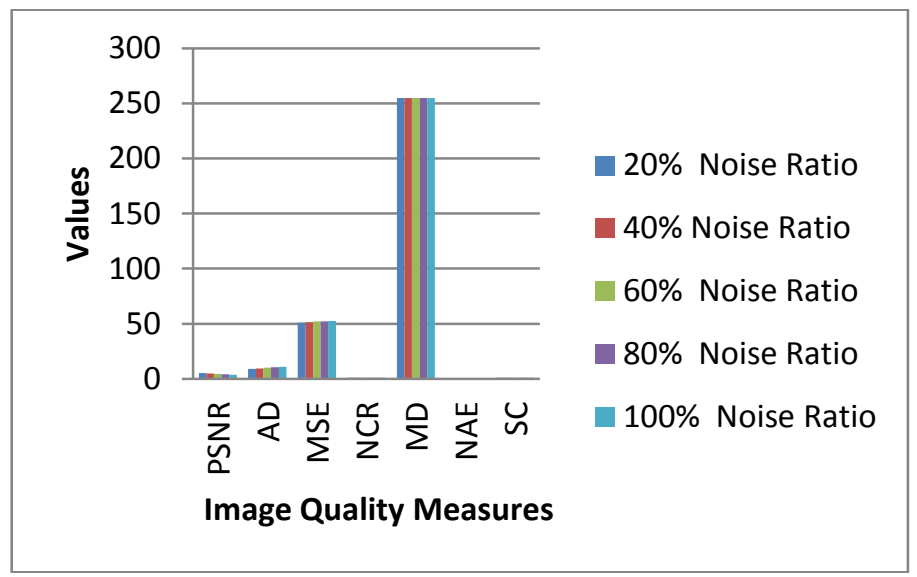

Fig 2: Graphical Representation of Image Quality Measures at different Gaussian noise levels for Average Filter 
Table 1: Image Quality Measures for Gaussian noise

\begin{tabular}{|c|c|c|c|c|c|c|c|}
\hline Filter & PSNR & AD & MSE & NCR & MD & NAE & SC \\
\hline $\begin{array}{c}\text { MEDI } \\
\text { AN }\end{array}$ & $\begin{array}{c}23.934 \\
4\end{array}$ & 2.2539 & $\begin{array}{c}21.001 \\
9\end{array}$ & 1.0000 & 255 & 0.0101 & 1 \\
\hline $\begin{array}{c}\text { AVE } \\
\text { RAG } \\
\text { E }\end{array}$ & 12.5 & 6.85 & 51.41 & 1 & 164 & 0.03 & 1 \\
\hline $\begin{array}{l}\text { WIE } \\
\text { NER }\end{array}$ & 20.89 & 3.27 & 26.52 & 1 & 42 & 0.01 & 1 \\
\hline $\begin{array}{c}\text { LAPL } \\
\text { ACIA } \\
\mathbf{N}\end{array}$ & -15.14 & 208.1 & 252.59 & 0.4 & 255 & 0.94 & 3.12 \\
\hline $\begin{array}{c}\text { CAN } \\
\text { NY }\end{array}$ & -17.6 & 211.25 & 254.99 & 0.05 & 255 & 1 & $\begin{array}{c}3.80 \mathrm{E} \\
+003\end{array}$ \\
\hline $\begin{array}{c}\text { PRE } \\
\text { WITT }\end{array}$ & -17.68 & 211.27 & 254.99 & 0.04 & 255 & 1 & $\begin{array}{c}4.93 \mathrm{E} \\
+003\end{array}$ \\
\hline $\begin{array}{l}\text { ROB } \\
\text { ERT }\end{array}$ & -17.68 & 211.2 & 254.99 & 0.03 & 255 & 1 & $\begin{array}{r}1.16 \mathrm{E} \\
+004\end{array}$ \\
\hline
\end{tabular}

Table 2: Image Quality Measures for Poisson noise

\begin{tabular}{|c|c|c|c|c|c|c|c|}
\hline Filter & PSNR & AD & MSE & NCR & MD & NAE & SC \\
\hline $\begin{array}{c}\text { MEDI } \\
\text { AN }\end{array}$ & 22.17 & 2.76 & 27.01 & 1 & 255 & 0.01 & 1 \\
\hline $\begin{array}{c}\text { AVER } \\
\text { AGE }\end{array}$ & 11.96 & 7.61 & 59.35 & 1 & 165 & 0.03 & 1 \\
\hline $\begin{array}{c}\text { WIEN } \\
\text { ER }\end{array}$ & 19.05 & 4.05 & 36.96 & 1 & 45 & 0.02 & 1 \\
\hline $\begin{array}{c}\text { LAPL } \\
\text { ACIA } \\
\text { N }\end{array}$ & -14.95 & 203.65 & 252.29 & 0.49 & 255 & 0.92 & 2.91 \\
\hline $\begin{array}{c}\text { ROB } \\
\text { YITT }\end{array}$ & -17.7 & 213.64 & 255 & 0.04 & 255 & 1 & $4.51 E+$ \\
\hline $\begin{array}{c}\text { PRE } \\
\text { CANN }\end{array}$ & -17.7 & 213.63 & 255 & 0.05 & 255 & 0.92 & $3.90 E+$ \\
\hline $\begin{array}{c}\text { Y } \\
\text { RI }\end{array}$ & 213.66 & 255 & 0.03 & 255 & 1 & $6.55 E+$ \\
& & & & & & 003 \\
\hline
\end{tabular}

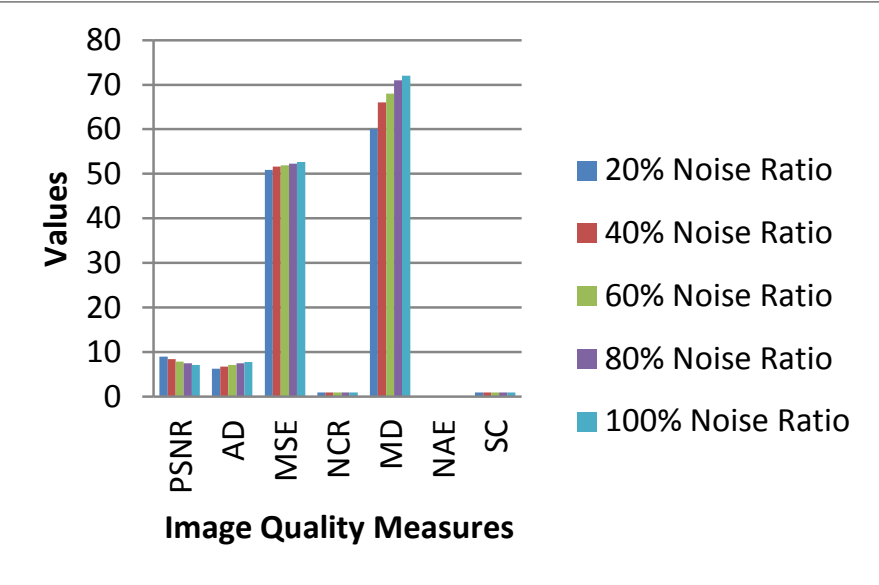

Fig 3: Graphical Representation of Image Quality Measures at different Gaussian noise levels for Wiener Filter 


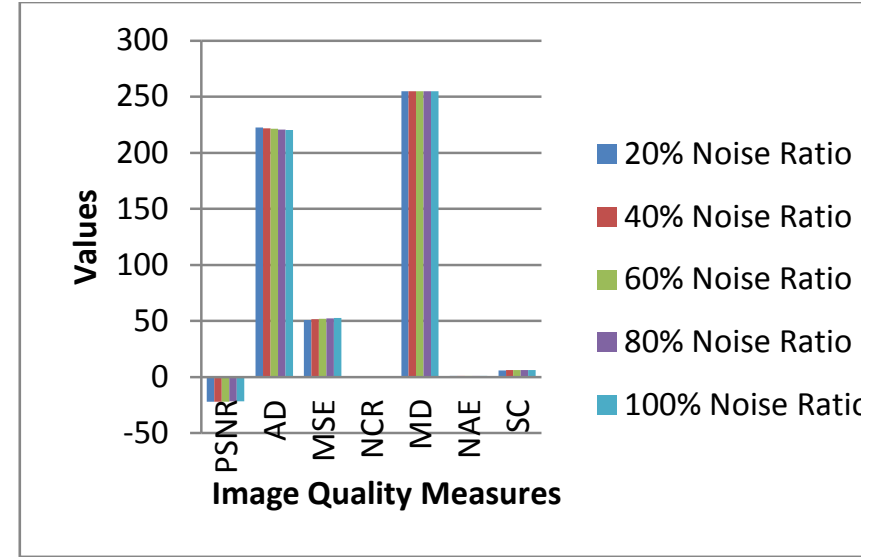

Fig 4: Graphical Representation of Image Quality Measures at different Gaussian noise levels for Prewitt Filter

Table 3: Image Quality Measures for Salt and pepper noise

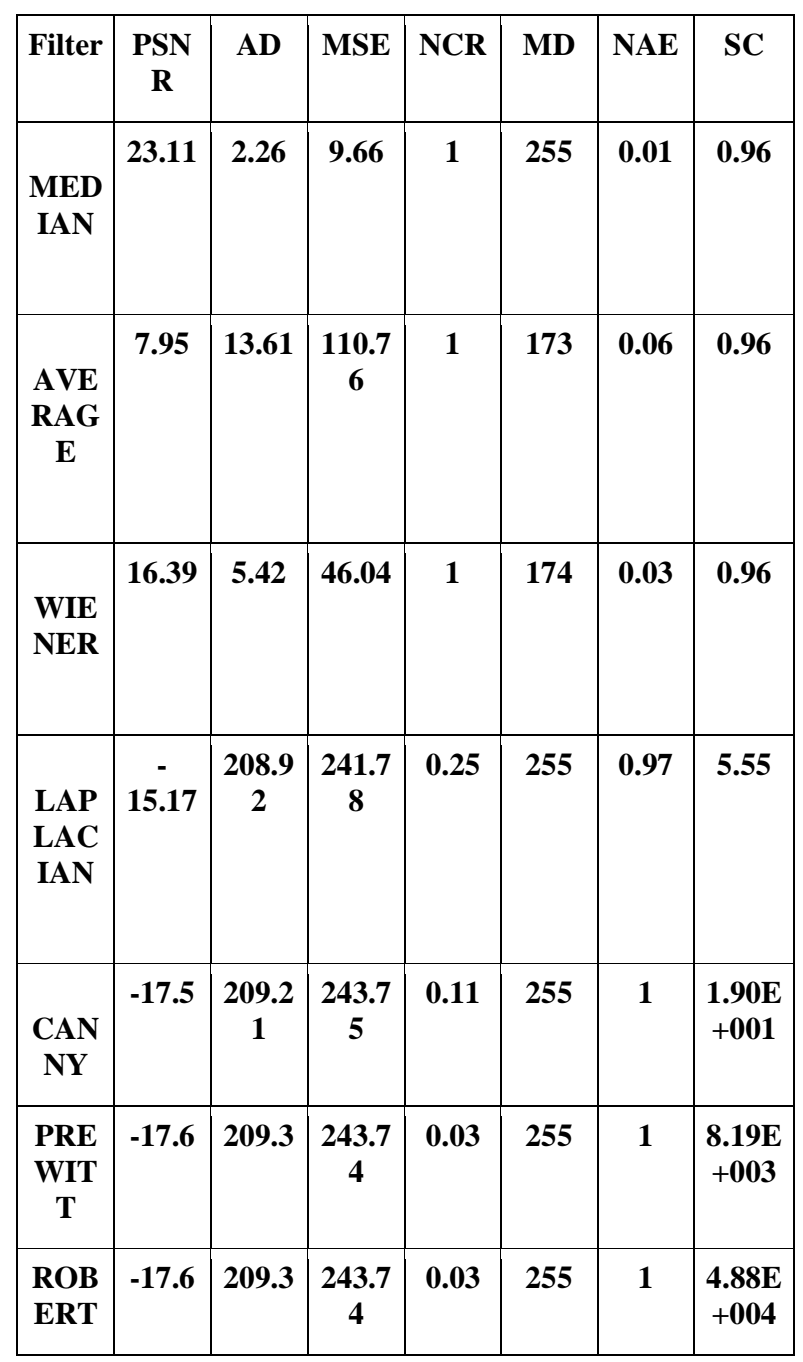

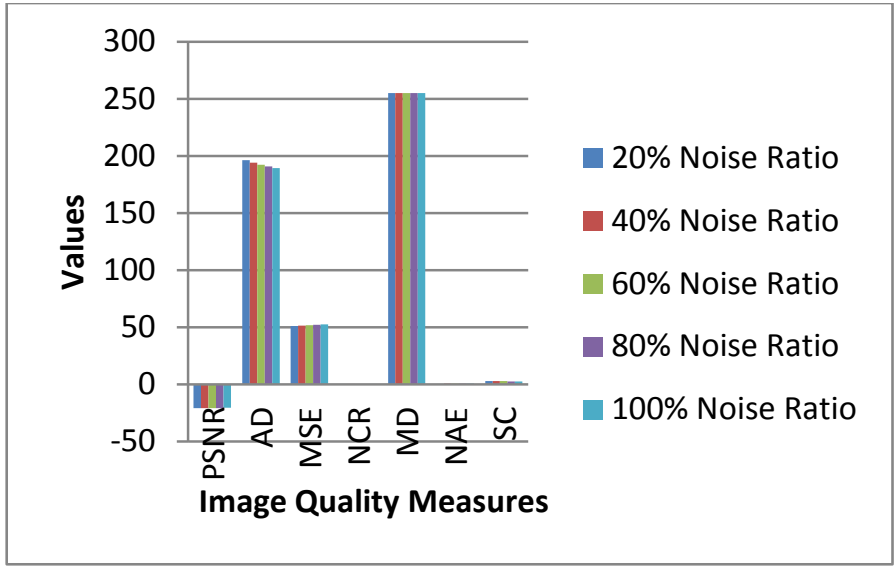

Fig 5: Graphical Representation of Image Quality Measures at different Gaussian noise levels for Laplacian Filter

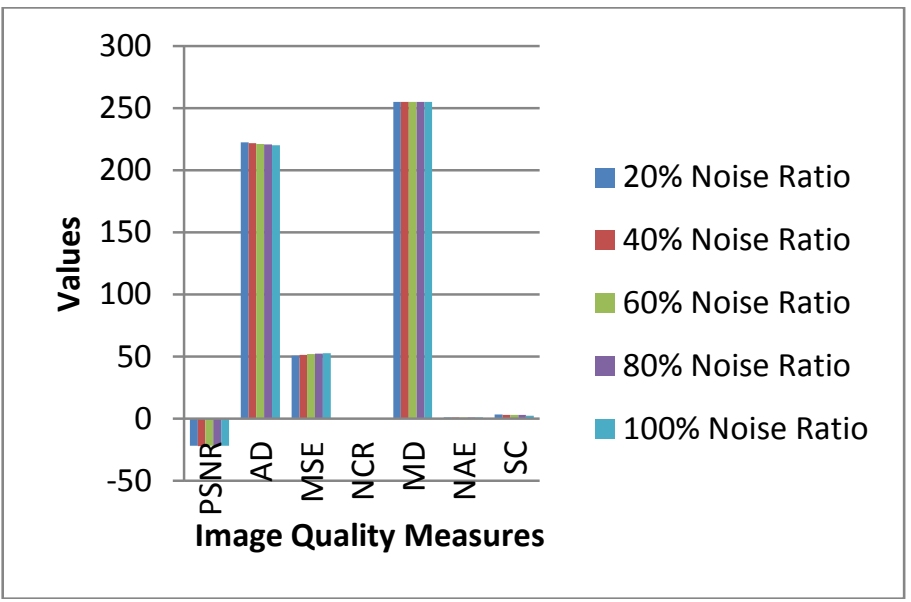

Fig 6: Graphical Representation of Image Quality Measures at different Gaussian noise levels for Canny Filter

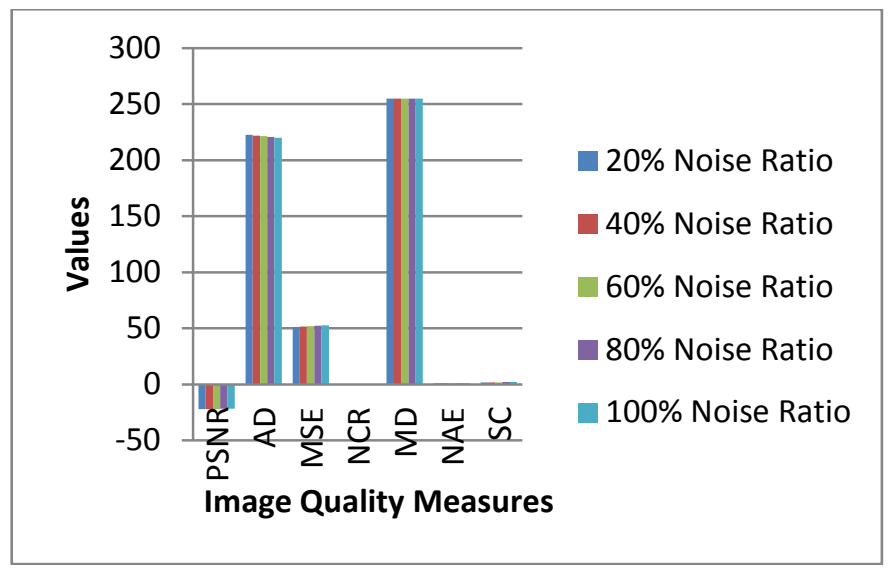

Fig 7: Graphical Representation of Image Quality Measures at different Gaussian noise levels for Robert Filter 
Table 4: Image Quality Measures for Speckle noise

\begin{tabular}{|c|c|c|c|c|c|c|c|}
\hline Filter & PSNR & AD & MSE & NCR & MD & NAE & SC \\
\hline $\begin{array}{c}\text { MEDI } \\
\text { AN }\end{array}$ & 18.55 & 4.1 & 50.39 & 1 & 255 & 0.03 & 1 \\
\hline $\begin{array}{c}\text { AVE } \\
\text { RAG } \\
\text { E }\end{array}$ & 9.41 & 11.39 & 107.59 & 1 & 168 & 0.05 & 1 \\
\hline $\begin{array}{c}\text { WIEN } \\
\text { ER }\end{array}$ & 13.33 & 7.84 & 88.41 & 1 & 63 & 0.04 & 1 \\
\hline $\begin{array}{c}\text { LAPL } \\
\text { ACIA } \\
\mathbf{N}\end{array}$ & -14.03 & 183.24 & 246.24 & 0.42 & 255 & 0.85 & 2.55 \\
\hline $\begin{array}{c}\text { CAN } \\
\text { NY }\end{array}$ & -17.59 & 208.18 & 255 & 0.05 & 255 & 1 & $\begin{array}{c}3.88 E \\
+003\end{array}$ \\
\hline $\begin{array}{c}\text { PRE } \\
\text { WITT }\end{array}$ & -17.55 & 208.21 & 255 & 0.04 & 255 & 1 & $\begin{array}{c}5.15 E \\
+003\end{array}$ \\
\hline $\begin{array}{l}\text { ROB } \\
\text { ERT }\end{array}$ & -17.55 & 208.24 & 255 & 0.01 & 255 & 1 & $\begin{array}{c}1.50 \mathrm{E} \\
+004\end{array}$ \\
\hline
\end{tabular}

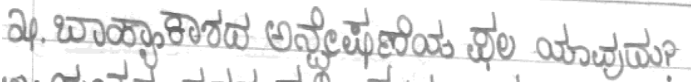

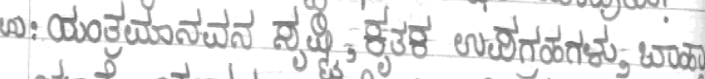

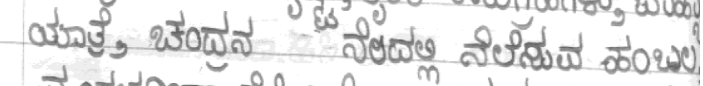

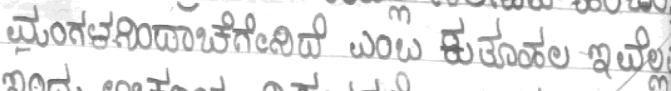

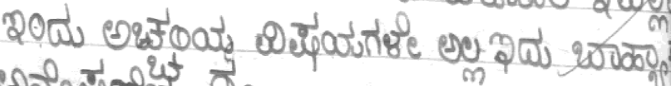

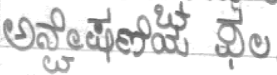

Fig 8(a): Binarized image of Original image

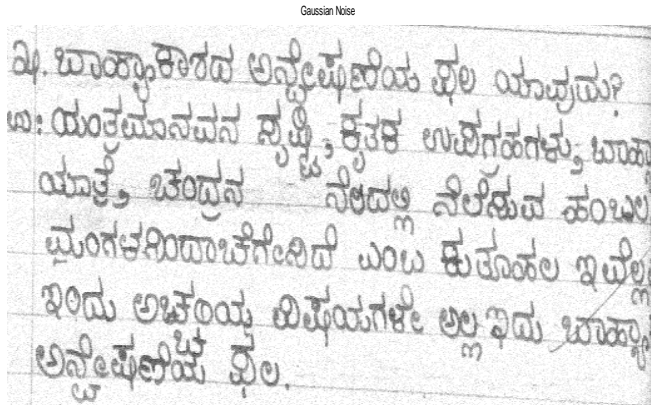

(b) Binarized image added with Gaussian Noise

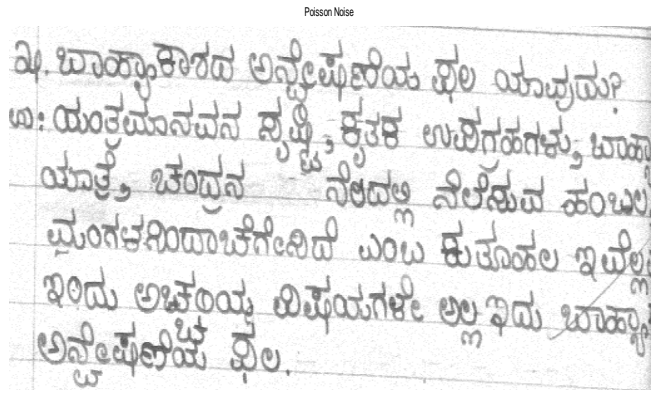

(c) Binarized image added with Poisson Noise

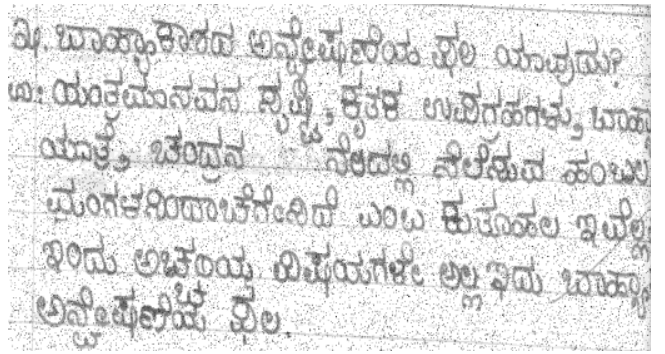

(d) Binarized image added withSalt and pepper Noise

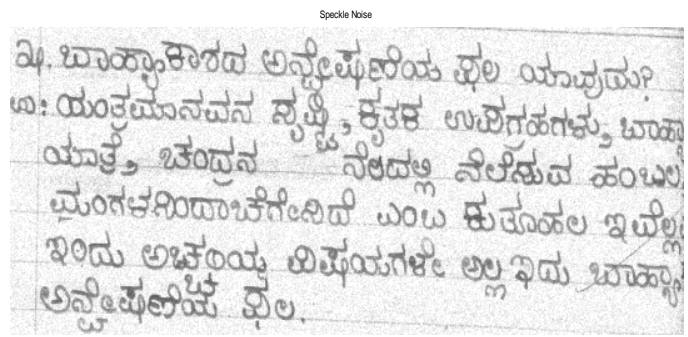

e) Binarized image added with Speckle Noise 


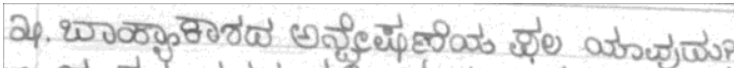

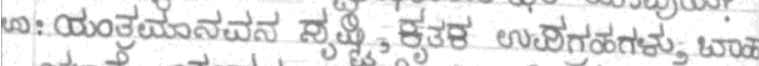

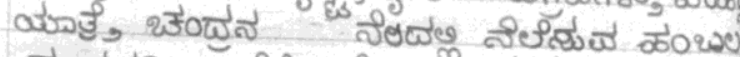

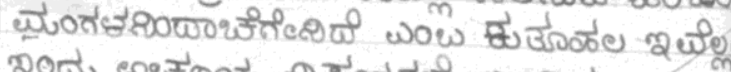
20దు అశ్రయ్

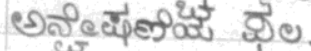

\section{Gaussian noise}

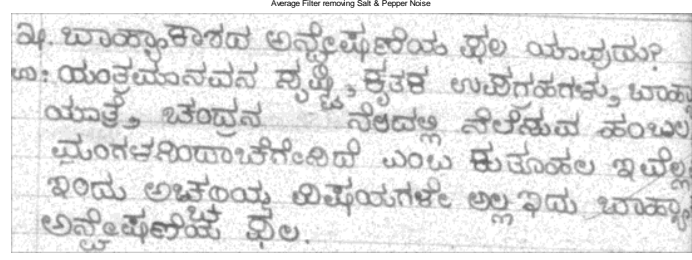

\section{Salt and Pepper noise}

Fig 9: Average Filter removing the 4 types of Noise

\section{Fig 10: Median filter removing the 4 types of Noise}

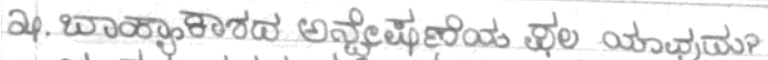

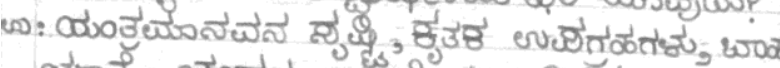

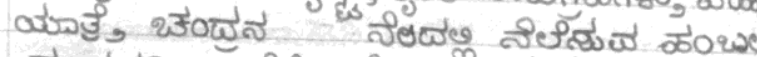

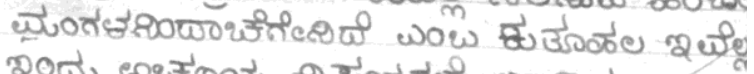

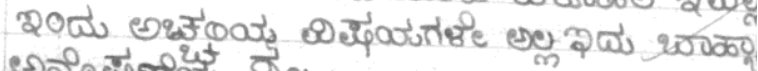

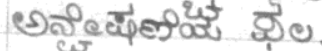

\section{Gaussian noise}

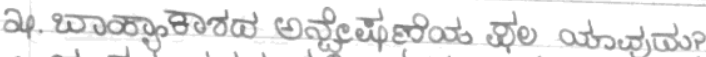

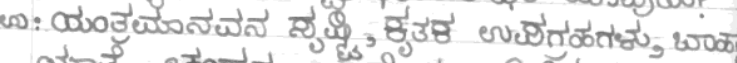

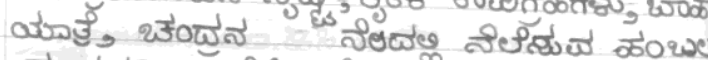

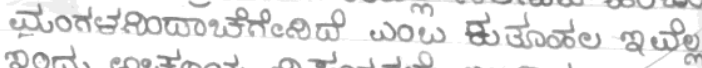

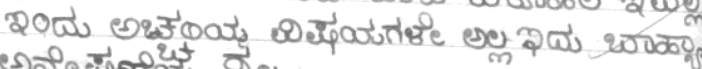

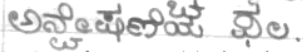

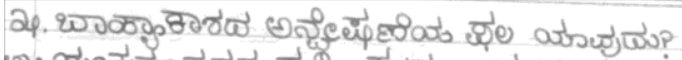

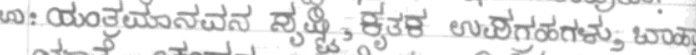

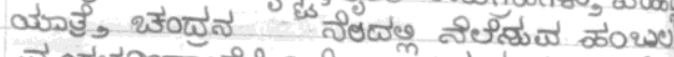

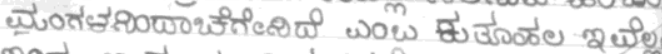

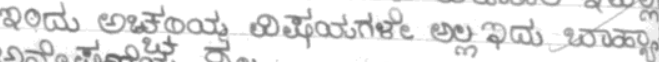

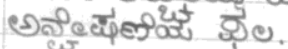

\section{Poisson noise}

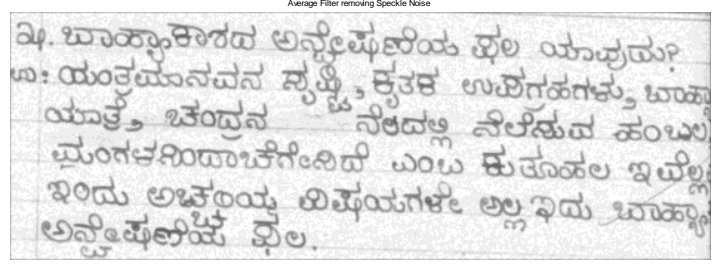

Speckle noise

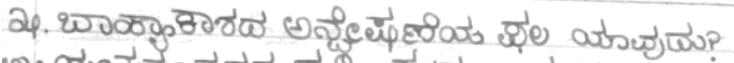

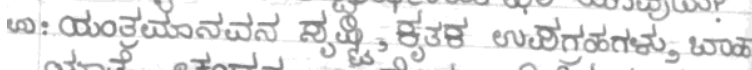

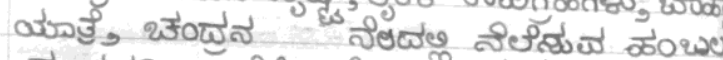

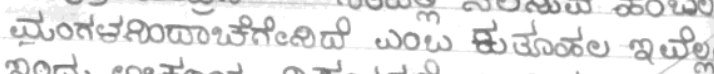

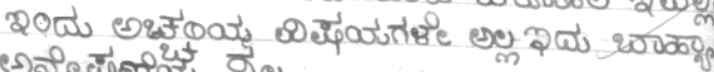

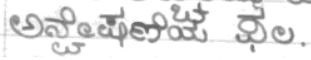

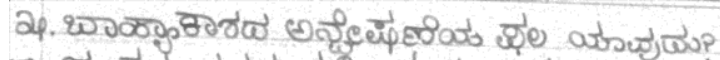

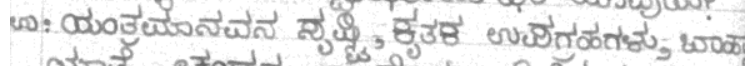

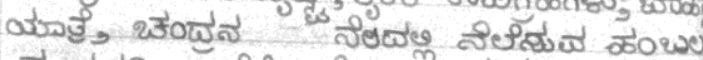

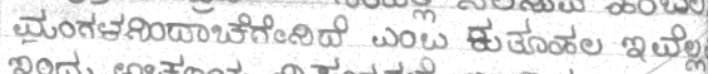

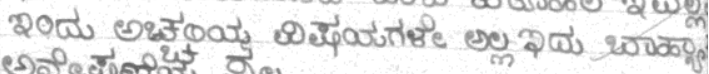

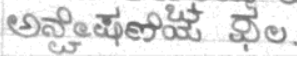

speckle noise 